\title{
Fondements historiques du principe de la reconstruction en chirurgie vasculaire
}

Par Charles Hahn

La plupart d'entre nous, chirurgiens cardiaques, vasculaires ou cardiovasculaires, avons commencé notre carrière professionnelle en acquérant des notions de chirurgie générale puis de chirurgie thoracique ou d'une autre spécialité médicale ou chirurgicale. A l'époque de notre formation, la philosophie générale de la chirurgie consistait en l'exérèse totale ou partielle des organes atteints. Cette chirurgie destructrice est très ancienne: elle est illustrée par le martyre de Ste Agathe qui, au début de la chrétienté subit une amputation des deux seins, à titre punitif. Dans une période plus récente et pour des indications plus défendables, on trouve une des premières descriptions, en 1634, des différentes étapes d'une ablation chirurgicale d'un sein cancéreux par Peter Lowe. Dans le domaine des mutilations périphériques, particulièrement des amputations de jambe, le $16^{\mathrm{e}}$ siècle est riche de très beaux documents illustrant la dextérité des chirurgiens comme Von Gersdorf de Strassbourg qui, en 1540, amputait des jambes sans anesthésie en un temps record. Cette technique s'est propagée à travers les siècles, mais heureusement, dès décembre 1846, l'intervention pouvait s'effectuer sous anesthésie grâce au travail de Liston. Qu'on nous permette de rappeler, à propos de l'anesthésie, que ce procédé a été fort bien défini par Ilza Veith, la grande historienne de la médecine, comme étant: «Ce cadeau sans prix qui permet d'éliminer les horreurs de la douleur.» Si l'on ne perd pas de vue une certaine vision internationale du développement de la chirurgie, on peut considérer que l'anesthésie est pratiquement entièrement une contribution américaine au développement de la chirurgie, développement qui se fit entre 1842 et 1846.

A propos de l'amputation de jambes, il n'est pas sans intérêt de relever que Pott, autour de 1780, consacra un traité à prouver la nécessité des amputations. Comme cela arrive souvent dans notre profession, deux autres traités parurent, l'un par un médecin de l'armée prussienne, l'autre par un de nos compatriotes lausannois, le $\mathrm{D}^{\mathrm{r}}$ Tissot, visant à démontrer que les amputations étaient toujours inutiles. Un langage qui est resté d'actualité car il n'est pas une affection cardiaque ou vasculaire, congénitale ou acquise, qui ne soit l'objet de prises de positions partisanes. La réponse de Pott à ces 
deux auteurs est un exemple de courtoisie et de sincérité: «Toutefois, si nous pouvons admettre que la doctrine défendue par ces gentlemen soit le produit de considérations humaines, leur donnant la conviction de leur bien-fondé, nous devons les féliciter de leurs intentions, bien que nous ne puissions accepter leur jugement.» Cette réponse pourrait être utilisée sans modification dans les discussions que nous avons sans cesse au sujet de la chirurgie cardiaque ou vasculaire.

Considérés comme des barbiers ou des chirurgiens - le terme approprié dépendant de considérations géographiques et historiques -, nous eûmes à affronter constamment le conservatisme de nos confrères médecins, même si la plus grande partie de nos efforts visait à n'utiliser la chirurgie que lorsqu'elle était absolument nécessaire, tout en cherchant de plus en plus activement et fréquemment, chaque fois que cela s'avérait possible, à remplacer des mutilations par des réparations.

Cette attitude a été largement appliquée au cours de ces 40 dernières années. En matière de chirurgie du poumon, notre génération vécut l'époque de l'éradication de la tuberculose pulmonaire par une approche combinée médicale et chirurgicale. L'indication aux résections pulmonaires n'intervenait qu'une fois le traitement médical correctement appliqué et seulement pour liquider les lésions restantes. Actuellement, les exérèses pulmonaires pour tuberculose sont rares en raison de la qualité du traitement médical et surtout de la qualité de la prévention. Au début, l'alternative à cette tactique chirurgicale consistait à placer des malades en sanatorium pour des années, ce qui, par la mutilation de la qualité de leur vie, laissait souvent des séquelles graves aussi bien sur le plan psychologique que sur le plan physique.

De tels exemples peuvent être multipliés à l'infini. Dans la chirurgie gastrique ou gastro-duodénale, nous vécûmes la période où les résections d'estomac pour ulcère gastro-duodénal constituaient la base des programmes opératoires. Mutilations du tractus gastro-intestinal dont les indications sont devenues très rares en raison de l'amélioration de la compréhension de la maladie ulcéreuse, de son meilleur traitement médical ou d'une chirurgie non mutilante (vagotomies). Et que dire de la lithiase urinaire qui donna tant d'importance pendant des siècles aux lithotomistes!

La philosophie réparatrice en chirurgie cardiovasculaire peut être illustrée par le développement du traitement des plaies artérielles. Dans une première étape, le but était de sauver la vie des malades en liant les artères blessées. Puis en traitant les artères malades, en mettant au point différentes 
techniques destinées à la réparation des vaisseaux et, par conséquent, à la préservation des organes ou tissus qu'ils irriguent.

Rappelons encore une fois que nos racines plongent profondément dans notre passé. Si nous prenons les ligatures pour exemple, on sait que l'homme préhistorique connaissait l'utilisation de fibres végétales ou animales pour arrêter les hémorragies des vaisseaux blessés. Il utilisait généralement des aiguilles faites à base d'os. Dans la Grèce antique, il était courant d'utiliser du «catgut» dans la construction des harpes et autres instruments de musique. Par la suite, ce matériel, connu, fut utilisé par Rhazes et Albucasis au courant du $10^{\mathrm{e}}$ siècle. Mais déjà au $2^{\mathrm{e}}$ siècle après Jésus-Christ, Galien utilisait la soie pour la ligature des vaisseaux. Autrement dit, le plus élémentaire des procédés chirurgicaux appliqués au système cardiovasculaire date du début de l'ère chrétienne et non pas du Moyen Age comme il est courant de le dire. Les problèmes principaux posés par les ligatures artérielles étaient liés à l'absence totale d'asepsie ou d'antisepsie, d'où de graves complications, telles des hémorragies secondaires quelquefois mortelles ou des complications ischémiques pouvant conduire à des gangrènes. La peur de l'élimination du matériel de suture, suite d'infections, conduisit à l'utilisation de plus en plus courante du cautère - fer chaud la plupart du temps. Ce n'est qu'à partir du moment où Lister institua l'antisepsie que le matériel de suture revint en force dans les usages chirurgicaux. C'est à cette époque que les chirurgiens apprirent à ne plus humecter le matériel de suture dans leur bouche avant de l'enfiler dans le chat de l'aiguille, selon les habitudes ancestrales de la couture.

Il fallut plus de vingt ans pour que les médecins acceptent les idées de Lister.

A propos de la réparation des artères, il faut souligner l'importance des conflits militaires dans le perfectionnement de leur traitement. Dans la vie civile, la saignée a longtemps été le traitement spécifique de la plupart des maladies. Certaines se compliquaient d'hémorragies graves par blessures accidentelles de l'artère brachiale. Ce n'est qu'en 1769 que Hallowel rapporta un cas opéré par Lambert de réparation d'une artère humérale blessée au cours d'une saignée. Par la suite d'autres cas de réparations artérielles furent rapportés par Eck et Postempski, ce qui entraîna une énorme stimulation aussi bien clinique qu'expérimentale. C'est à cet enthousiasme que l'on dut quantités de techniques destinées à la reconstitution d'artères: interposition d'une tube de verre fin selon la méthode de Abbe; les anastomoses directes termino-terminales de Briau et Jaboulay; les dif- 
férentes techniques mises au point dès 1896 par Murphy (résection d'un segment d'artère fémorale déchiqueté et rétablissement de la continuité par anastomose directe, ou sa méthode d'invagination). C'est à la même période que d'autres auteurs décrivirent d'ingénieuses techniques dont la méthode de la suture continue par surjet utilisée par Dorance au début de ce siècle. Mais c'est bien sûr à l'énorme travail de Carrel et de Guthrie que l'on doit l'affinement et la méticulosité des techniques d'anastomose. Carrel était convaincu, dès 1906, que le moindre dommage à l'endothélium se soldait par la formation d'une thrombose; la technique des sutures artérielles devait donc être axée sur la prévention de lésions de l'intima. D'où une préparation soigneuse de l'artère en prévenant toute lésion de sa paroi par des clamps ou du matériel de suture trop grossier. Carrel eut aussi l'idée de patcher les artériotomies par du matériel biologique, qu'il s'agisse de veines ou de péritoine. Il y a donc plus de 80 ans que la démonstration a été faite que ce matériel d'apparence fragile ne donnait pas, en tout cas dans la majorité des cas, de formations anévrismales, même s'il était interposé sur des vaisseaux soumis à de hautes pressions. Ces différentes méthodes mirent un terme à l'utilisation du cautère qu'il se soit agi de fer chauffé au rouge, d'huile bouillante accompagnée ou non de poudre magique, comme la poudre de sympathie décrite par R. White!

Cet énorme travail expérimental destiné à la réparation des artères et à la restauration d'une circulation normale mit un temps infini pour passer en usage clinique. Encore une fois, ce sont les guerres qui permirent la standardisation du traitement chirurgical des artères lésées. Pourtant, son application resta limitée durant la deuxième guerre mondiale; il devint effectif pendant la guerre de Corée et celle d'Indochine, limitant le nombre des mutilations.

Dans la pratique civile, je n'oublierai jamais le cas d'un jeune homme de 20 ans qui, en 1947, subit une amputation des deux jambes à la suite de lacérations des artères fémorales que quelques sutures auraient pu sauver.

Un énorme pas en avant a été franchi avec le développement des prothèses vasculaires, après utilisation d'homogreffes pour remplacer la bifurcation aortique, comme l'a fait Oudot en 1950 pour une maladie de Leriche, puis Dubost en mars 1951 pour un anévrisme aortique. Le déséquilibre entre les besoins et les possibilités de conservation d'homogreffes dans des banques artérielles ne fit que s'accroître. Même si comme nous l'avons fait à cette époque, nos homogreffes étaient lyophilisées, donc conservables longtemps. Cela conduisit inévitablement au développement de prothèses 
artérielles. Quantités de matériaux différents furent utilisés jusqu'à ce que l'unanimité se fasse sur des prothèses de dacron qui apparurent comme un des meilleurs moyens de substitution ou de reconstruction artérielle. Les mêmes problèmes se posent actuellement en matière de remplacement cardiaque, soit par des homogreffes soit par des prothèses cardiaques (cœur artificiel).

La contribution française à la reconstruction artérielle, moyen de prévenir des mutilations, n'a pas été limitée à Oudot et à Dubost. Une des grandes contributions a été celle de Kunlin qui, en 1948, eut l'idée de ponter les oblitérations artérielles périphériques au moyen de veines saphènes. Si l'on considère que 2 ans auparavant Dos Santos avait mis au point la technique de désoblitération artérielle, celle du pontage de Kunlin apportait au chirurgien, avec les remplacements artériels au moyen d'homogreffes ou de prothèses, une panoplie chirurgicale complète.

Les anévrismes ont posé à des générations de chirurgiens des problèmes qui ne manquent pas d'intérêt. La première description du traitement chirurgical d'une telle lésion localisée dans un bras a été donnée au $\sigma^{\mathrm{e}}$ siècle après Jésus-Christ par Aëtius. Sa description se résume de la manière suivante: «Le vaisseau ayant été soigneusement libéré des tissus avoisinant et soulevé au moyen d'un crochet à l'extrémité mousse, les deux parties, proximale et distale, sont liées tandis que l'anévrisme est sectionné entre ces deux ligatures. La cavité anévrismale peut alors être laissée ouverte sans danger.» Dix siècles plus tard, Ambroise Paré donna une description chirurgicale très voisine de celle de Aëtius. Mais déjà au courant du $7^{\mathrm{e}}$ siècle après J.-C., Paul d'Aegina étendait les indications chirurgicales au traitement des anévrismes du cou et des régions articulaires.

Il est généralement admis que grâce à la période arabe, la connaissance médicale revint en Europe, à travers l'Espagne et l'Italie. L'Espagne tout particulièrement. Pendant cette période, Haly Abbas, un Persan, ouvrait les anévrismes longitudinalement après avoir lié l'artère en amont et en aval. Le procédé se généralisa en Espagne et fut utilisé largement par Albucasis et Averroes de Cordoue et Avenzohar de Seville. Cette méthode était appelée le procédé d'Antyllus.

Il n'est pas rare dans notre profession d'attacher une grande importance aux priorités. Grâce à un excellent travail de Broca, cité par Jean Natali, on peut apprendre que la première ligature attribuée à Hunter avait été pratiquée 6 mois plus tôt par Desault, en 1785. Hunter apprit cette technique grâce à un visiteur qui se trouvait chez Desault au moment de cette 
opération. Cela ne diminue aucunement le mérite de John Hunter qui, à côté de son intérêt pour les anévrismes, est connu pour son courage puisqu'il s'inocula, par des voies non naturelles, une blennorragie et une syphilis pour mieux étudier l'évolution de ces maladies sur sa propre personne. Longtemps plus tard, cette auto-expérimentation préluda aux premiers cathétérismes cardiaques.

Chacun, dans notre profession, doit beaucoup à ceux qui l'ont précédé. Je ne citerai qu'un exemple: celui de la découverte de la circulation du sang par W. Harvey, en 1628, découverte qui n'aurait probablement pas été possible sans le travail de base fait par son chef Fabricius de Aquapendente de Padoue.

Le traitement chirurgical des anévrismes de l'aorte et des grand vaisseaux, si riche en idées, illustre également certains traits psychologiques propres aux chirurgiens. Qu'on me permette de citer Mott qui, en 1829, à propos du cas d'un anévrisme d'une artère innominée opérée l'année précédente chez un homme de 57 ans, écrivait: «interrompre subitement le quart du débit cardiaque si près du cœur paraissait impossible à tout chirurgien tant les effets secondaires devaient être considérés, à priori, comme très importants. C'est la raison pour laquelle j'ai serré la ligature graduellement, mes yeux fixés sur la face du malade, prêt à la retirer si des symptômes alarmants étaient apparus. Mais, au lieu de cela, comme il ne montra aucun signe d'agitation du corps, mon plaisir fut immense.»

Pour compléter cette histoire, ajoutons que ce malade a été traité avec du vin dans l'idée d'activer la circulation - une pinte et demie de vin de Madère en l'espace de 24 heures. Toutefois, malgré ce traitement postopératoire parfait, le patient mourait au $26^{\mathrm{e}}$ jour en raison d'une hémorragie provenant d'une ulcération nécrotique de la blessure avec élimination des ligatures.

Sans entrer dans le détail d'autres procédés utilisés à l'époque, comme la galvanoplastie, la compression, le wiring, le wrapping, on peut admettre que la ligature, spécialement dans certaines situations anatomiques, permet une fibrose de l'anévrisme, éloignant la risque de rupture. Mais cela n'était pas une méthode reconstructive: la première qui puisse réellement mériter cet adjectif a été l'endoaneurysmoraphie de Matas qui date d'un siècle. Comme nous l'avons déjà dit, la première résection d'un anévrisme de l'aorte abdominale est due à Dubost qui la pratiqua au printemps 1951. Cette méthode ne nécessitait aucune technique spéciale de protection du cerveau, des reins, du foie ou du myocarde. Par contre, l'extension des résections 
d'anévrismes au reste de l'aorte impliqua le développement de méthodes de protection des organes momentanément privés de leur circulation.

Une des méthodes accessoires qui a joué un rôle capital dans le développement de la chirurgie cardiovasculaire a été la transfusion sanguine. La création de banques de sang a constitué une étape déterminante dans l'application de méthodes modernes. Ce sujet mériterait un important développement pour lui seul. C'est pourquoi je me contenterai de mentionner que la première transfusion animale a été faite en 1655 par Richard Lower. Elle a été administrée dans la veine d'un chien à partir de l'artère d'un autre, au moyen d'un tube d'argent. Le chien transfusé ne présenta aucun problème. C'est la raison pour laquelle, un peu plus tard, alors que la saignée paraissait un procédé thérapeutique d'autant plus efficace qu'elle était plus poussée - ce qui conduisit à de véritables exsanguinations - il apparut nécessaire de rétablir des conditions hémodynamiques de base par une transfusion!

La transfusion de sang devint une réalité à partir du moment où les groupes sanguins furent découverts et qu'on trouva les moyens de conserver ce sang. La possibilité d'opérer des anévrismes, quelles qu'aient été leur situation et leur étendue, se généralisa grâce au développement de la radiologie (Röntgen 1895), puis plus tard, dans les années 20, par la mise au point des artériographies. Le développement de l'anesthésie, des soins intensifs et de certaines techniques empruntées à la chirurgie à cœur ouvert permit des opérations de plus en plus sûres. La tactique chirurgicale consistant à libérer l'anévrisme posa des problèmes tant qu'on ne tint pas compte des idées de Matas. Ces dernières furent réanimées tout spécialement par l'école de Houston: au lieu d'isoler l'anévrisme, celui-ci fut simplement ouvert, la circulation étant restaurée par l'interposition à l'intérieur du sac anévrismal d'un substitut artériel. Une simplification considérable conduisant à des interventions plus rapides et plus sûres.

La plupart des anévrismes sont d'origine artériosclérotique, même si le my the d'une origine syphilitique continue à persister dans tous les traités. A côté de cette forme anévrismale, l'artériosclérose peut être la cause de sténoses ou d'oblitérations artérielles qui peuvent être localisées n'importe où, mais qui, de façon surprenante, sont souvent limitées dans leur extension. Si nous nous attachons à certaines localisations de ces lésions oblitératives, nous pouvons en tirer deux exemples qui illustrent bien notre philosophie visant à la protection de l'intégrité de l'organisme. C'est ainsi que si on considère les lésions aorto-iliaques connues depuis 1923 sous le nom 
de Maladie de Leriche, il s'agit là d'une localisation de la maladie artérioscléreuse qui conduit généralement, après quelques années, à des gangrènes des deux jambes et à d'autres complications. Avant l'heure de la chirurgie reconstructive, la seule alternative était la mutilation. Or, la plupart des patients affectés par cette lésion sont des gens jeunes. Leriche travaillait, à défaut d'autres moyens, sur le principe du développement d'une circulation collatérale par une sympathectomie péri-artérielle ou par une artériectomie. L'efficacité de ces méthodes était douteuse mais quelquefois donnait des résultats surprenants. En 1924, Royle introduisit la sympathectomie lombaire en tant que procédé électif permettant une dilatation périphérique, évitant la dénervation de vaisseaux dont Carrel avait démontré vingt ans plus tôt les effets négatifs. Les indications à la sympathectomie lombaire furent précisées par De Takats; ce fut le réel début de méthodes permettant une amélioration circulatoire diminuant de ce fait le nombre des mutilations.

Leriche, avec sa vision extraordinaire du futur, avait déjà écrit que l'idéal dans les occlusions artérielles serait de pouvoir attaquer la lésion directement afin de restaurer un débit normal dans le système vasculaire. Il doutait cependant que cela soit jamais réalisable mais changea d'idée lorsque ses élèves développèrent les endartériectomies, les pontages, puis les opérations directes. En lisant la description de l'opération faite par Oudot en $1950 \mathrm{chez}$ un malade de 51 ans, on est frappé de constater que ce chirurgien accompagnait sa résection abdominale par une endartériectomie complémentaire, proximale, de manière à obtenir une paroi aortique souple permettant la suture de l'homogreffe qui avait été conservée pendant trois semaines, grâce à la technique de Jean Faurel! Ce principe d'associer endartériectomie et un pontage ou une substitution artérielle, largement utilisé actuellement dans la chirurgie des lésions diffuses des artères coronaires, date donc de plus de 35 ans.

Ce qui est vrai pour les jambes est également vrai pour les bras. Grâce à la description donnée en 1864 par Raynaud (thèse sur l'asphyxie locale et la gangrène symétrique des extrémités) on savait qu'une gangrène des doigts pouvait se produire dans cette maladie et entraîner des mutilations. Une chirurgie directe n'entrait pas en ligne de compte dans ces affections. Par contre, les sympathectomies thoraciques s'avèrent une excellente méthode permettant la prévention de mutilations. La maladie de Raynaud est d'ailleurs souvent secondaire à une autre affection. Dans ces cas, c'est bien sûr le traitement causal qui permettra la guérison de la lésion. 
Le traitement chirurgical des anévrismes vise à prévenir des interventions d'urgence lors de ruptures spontanées. Ces opérations comportent un risque élevé. Les réparations artérielles dans des cas d'obstructions visent à prévenir des amputations de jambes, ou, dans les cas de lésions d'artères à destinée encéphalique, à éviter des accidents cérébraux graves, laissant souvent des séquelles durables.

En conclusion, on peut dire que l'étude du développement historique de certaines lésions du système cardiovasculaire apporte la preuve que nous pouvons actuellement éviter la plupart des désastres que des malades ont vécus avant l'époque de la chirurgie reconstructive. Cette philosophie visant à la protection de l'individu dans son intégrité est le produit de nombreux travaux dont les premiers datent de l'Antiquité. Mais seule la synthèse de connaissances provenant d'origines très différentes a permis de rationaliser un traitement chirurgical visant à prévenir des mutilations graves ou à en limiter l'extension.

\section{Summary}

The approach in arterial surgery was for centuries based on mutilation, as in most surgical procedures. It required a long period of time to develop sufficient knowledge to build other tactical approaches, based on new surgical techniques, in order to prevent the removal of organs or limbs, or to avoid severe neurological disturbances. This change in our ideas is illustrated in the following two examples: the repair of wounded arteries and the surgical treatment of arteriosclerotic lesions of the aorta and great vessels.

Prof. Charles Hahn

Centre de recherches cardiovasculaires

CH-1261 Arzier (VD) 